\title{
Nanoscale Temperature Measurements Using Phonon Scattering
}

\author{
M. J. Lagos ${ }^{1,2}$ and P. E. Batson ${ }^{2}$
}

${ }^{1 .}$ Department of Materials Science and Engineering, McMaster University, Hamilton, Ontario, Canada.

2. Department of Physics and Astronomy, Rutgers University, Piscataway, NJ.

The theoretical description of spontaneous and thermally-induced processes by fast electrons is usually presented in inelastic vibrational cross sections with the inclusion of pre-factor terms proportional to $(n+1)$ and $(n)$, for the phonon emission (loss) and absorption (gain) in bulk matter, respectively. The term " $n$ " represents the phonon occupational number and it depends on the probed system temperature. This implies that measurement of scattering probabilities through conventional electron spectroscopy methods can permit us access to sample temperature information. Also, as consequence of the principle of detailed balancing (PDB) the loss and gain scattering are linked through the Boltzmann factor at thermal equilibrium. Experimental validation of the loss-gain relationship was reported for large surfaces systems [1]. But, the exploration of spatially-resolved energy-gain processes induced by relativistic electrons in nanostructures is still in its infancy. In this work, we present a study of the phonon scattering in nanostructures as a function of temperature, through which we determined the local temperature of nanoobjects with high precision and sub-nanometer spatial resolution.

This study obtained EELS/EEGS spectra in nanostructures (nanoparticles, nanometric plates and interfaces), using the Nion UltraStem equipped with a monochromator to study the the vibrational response in the infrared range using a $\sim 1.5-2 \AA$ probe with energy spread of $\sim 9 \mathrm{meV}$, at $60 \mathrm{kV}$. Our instrument allows the detection and mapping of bulk and surface phonon excitations down to $20-30$ $\mathrm{meV}$ [2]. At room temperature, we were able to detect energy-gain and energy-loss signal because the scattering cross section for phonon emission are significant large, reaching between $0.1-1 \%$ of the zeroloss peak intensity in most cases. At higher temperatures, the inelastic phonon signal increases, with larger increases at lower excitation frequencies, as dictated by the statistical occupation number. We detected gain scattering in a large variety of nanostructures and Figure 1 illustrates these general findings, displaying spectroscopic curves acquired as a function of temperature in a single suspended $\mathrm{MgO}$ nanoparticle.

The background can be removed leaving the gain and loss phonon scattering signal (Fig. 2a). These can be used to measure the local temperature using PDB by plotting the logarithm of the ratio of the scattering intensities on the gain and loss sides of zero energy. Fig. $2 b$ illustrates this behavior. We verified that these RGL curves are linear for several nanomaterials, supporting the use of PDB on the nanoscale. Our experimental results obtained at room temperature indicate that the probed nanosystem indeed is close to $290 \mathrm{~K}$ with a precision of about $7-10 \mathrm{~K}$ with $\sim 99 \%$ of confidence level and agreed very well with the accepted values for room temperature conditions. Similar results were obtained for measurements at high temperatures. We found also that temperatures obtained from bulk phonon scattering compared well with those obtained using surface phonon polariton scattering, typically present in finite-size objects [3], suggesting that temperature gradients from the inside towards the surface should be detectable. We also investigated the localization degree of our measurements and we found that highly-localized scattering, associated with the excitation of short-wavelength acoustic phonons, can be used to perform measurements with high spatial resolution, because bulk scattering can vary drastically within neighboring atom columns (impact scattering). 
We show that the temperature of a nanosized volume of matter can be measured experimentally using the energy gain/loss scattering, verify that PDB holds for nanomaterials at thermal equilibrium. We think that our results will also stimulate investigations towards atomic-resolution temperature spectroscopy in nanostructures exploring different collection conditions. An outstanding question still remains about why polar insulators produce such good spatial resolution, while other recently examined systems produced poor lateral resolution. But we think this method holds much promise for characterization studies which require spatially-resolved thermal imaging capabilities and nanoscale thermal response (catalysis, nanoscale energy transport, etc.) [4].

\section{References:}

[1] H. Ibach, Physical Review Letters 24 (1970), p. 1416.

[2] M. J. Lagos et al, Nature 543 (2017), p. 529.

[3] M. J. Lagos et al, Microscopy (2018).

[4] This research was supported by the U.S. Department of Energy, Office of Science, Basic Energy Sciences under Award \# DE-SC0005132. M.L acknowledges G. Botton for providing financial support to present this work.

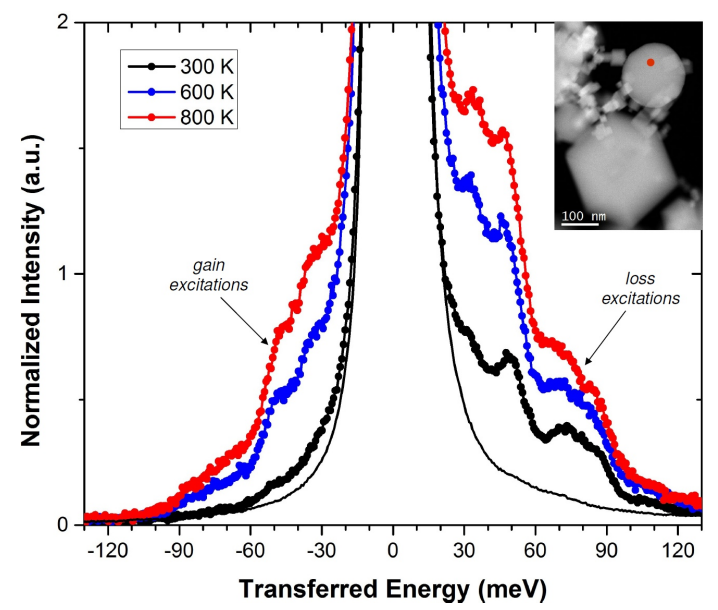

Figure 1. EELS/EEGS spectra acquired in a single $\mathrm{MgO}$ nanosphere as a function of temperature. Inset shows an ADF image of the probed particle. The red dot indicated the beam position. Note that the energy-loss/gain scattering signal shows up over the tails of the zero loss peak (continuous black curve).
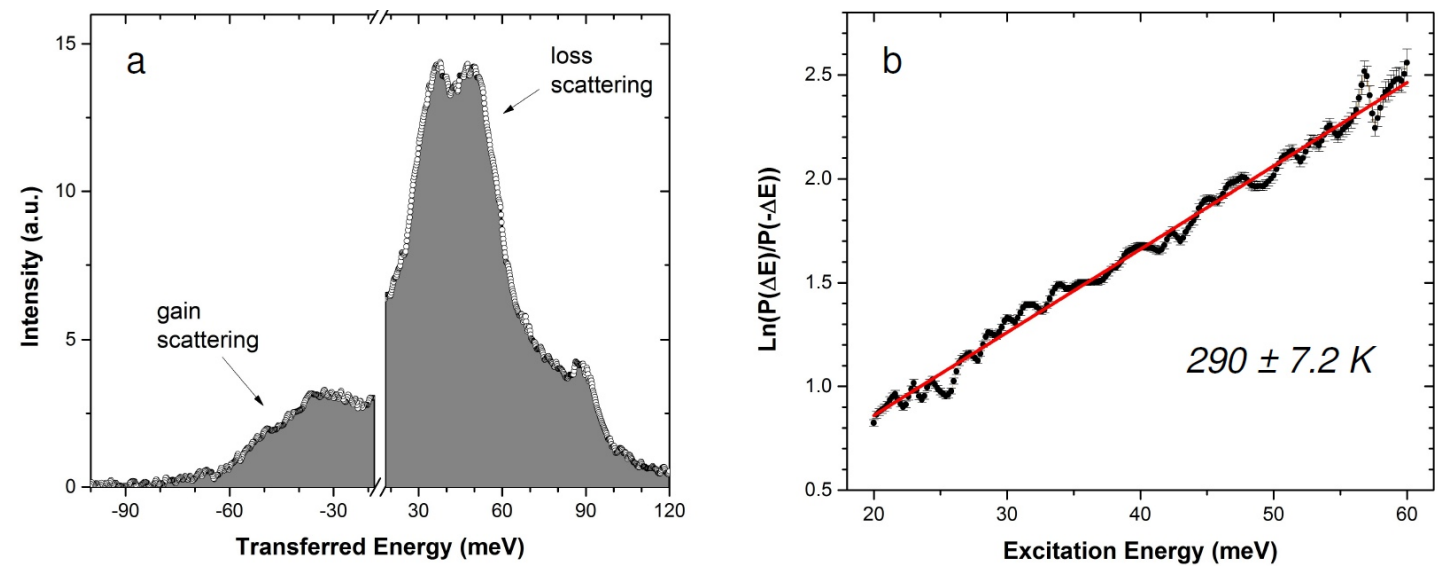

Figure 2. a) Gain and Loss scattering obtained from a single $\mathrm{MgO}$ nanocube at room temperature. b) Plot of the logarithm of the ratio between gain and loss scattering as a function of excitation energy. Note the linear behavior of the RGL curve. From the fit we obtained the local temperature of the single particle. 